\title{
Uma abordagem de otimização robusta no planejamento agregado de produção na indústria cítrica
}

\author{
José Renato Munhoz ${ }^{\mathrm{a} *}$, Reinaldo Morabito ${ }^{\mathrm{b}}$ \\ a*jose.munhoz@citrovita.com, Citrovita, Brasil \\ ${ }^{\mathrm{b}}$ morabito@ufscar.br, UFSCar, Brasil
}

\begin{abstract}
Resumo
Neste trabalho o planejamento agregado de produção de sucos concentrados congelados de laranja é modelado com considerações de incertezas em alguns parâmetros, de modo a constituir uma ferramenta efetiva de suporte à tomada de decisões. A abordagem de otimização robusta é baseada em um modelo determinístico de programação linear com múltiplos produtos, estágios e períodos proposto em Munhoz e Morabito (2010). Além das decisões de produção, mistura e estocagem de sucos, esse modelo também incorpora o planejamento de colheita da laranja, levando-se em consideração as curvas de maturação das frutas. Um estudo de caso foi realizado em uma empresa do setor localizada no estado de São Paulo, envolvendo várias plantas e uma rede de distribuição internacional. Os resultados computacionais obtidos com a abordagem de otimização robusta, utilizando uma linguagem de modelagem algébrica e um aplicativo de última geração de solução de problemas de programação matemática, indicam que a abordagem tem potencial para ser aplicada com sucesso em situações reais.
\end{abstract}

Palavras-chave

Otimização robusta. Problemas de planejamento de produção com múltiplos produtos, estágios e períodos. Programação linear. Suco concentrado congelado de laranja.

\section{Introdução}

0 desenvolvimento da indústria de suco de laranja ocorreu principalmente nos EUA na década de 1930, devido ao aumento do consumo do produto (VIEGAS et al., 1983). Em 1944 teve início a comercialização de suco concentrado congelado de laranja. No Brasil, as primeiras agroindústrias processadoras de suco de laranja começaram a operar no início dos anos 1960 (CASTILHO, 2006). A primeira fábrica de suco concentrado e congelado nos moldes americanos foi a Suconasa (Sucos Nacionais S.A.), implantada em Araraquara (SP), em 1963. Segundo Hasse (1987), foi praticamente um transplante: tinha causas, motivações, agentes, capital, tecnologia e mercado externos. Não era uma iniciativa completamente americana porque foi realizada principalmente por um porto-riquenho que era dono da Toddy do Brasil.
No início dos anos 1980, o Brasil já se tornava o maior produtor e exportador mundial de suco, ultrapassando os EUA (CASTILHO, 2006; VIEIRA, 1997). 0 Quadro 1 apresenta alguma terminologia utilizada na indústria cítrica e de interesse para o presente trabalho, a Figura 1 mostra a produção anual de laranja desde 1995 e a Figura 2 apresenta as exportações anuais de suco concentrado de laranja no mundo desde 1995 (note a destacada liderança do Brasil na produção e exportação desse produto). Segundo dados do Instituto Brasileiro de Geografia e Estatística - IBGE (2010), o nível de produção de laranjas em 2010 estava estimado em 18.896.324 t, o que indica a manutenção dos patamares de produção do Brasil indicados na Figura 1.

0 estado de São Paulo concentra 75\% da produção brasileira de laranja, sendo responsável 
por 95\% das exportações de suco de laranja do país (CASTILHO, 2006). Segundo Neves et al. (2004), $82 \%$ da produção paulista de laranja, em média, destinam-se ao processamento, e o suco de laranja concentrado congelado é o $17^{\circ}$ principal produto da pauta das exportações brasileiras, sendo responsável por 72\% das exportações citrícolas. Com um parque industrial constantemente atualizado e incorporando novas tecnologias, o Brasil tornou-se líder, tanto no processamento de citros para a produção de suco concentrado congelado, em termos de tecnologia, qualidade e custo, como em logística de distribuição em âmbito mundial.

Nessa indústria cítrica, o processo de obtenção de produtos finais a partir das matérias-primas disponiveis no mercado envolve uma grande quantidade de informações, e o processamento e análise dessas informações podem apoiar a empresa na geração de um diferencial competitivo, que é a excelência no gerenciamento integrado da cadeia de suprimentos. A coordenação da produção, estoques e transporte de matérias-primas (diferentes variedades de laranja), produtos intermediários (sucos concentrados de laranja

Quadro 1. Terminologia utilizada na indústria cítrica.

\begin{tabular}{|cl|}
\hline $\begin{array}{c}\text { Caixa de } \\
\text { fruta }\end{array}$ & Unidade de peso equivalente a 40,8 kg ou 90 libras \\
\hline Brix & $\begin{array}{l}\text { Refere-se a porcentagem de sólidos solúveis ou } \\
\text { açúcares e ácidos, sendo quantificado em graus brix } \\
\text { por um instrumento utilizado para medir o índice de } \\
\text { refração de soluções, chamado de refratômetro }\end{array}$ \\
\hline Acidez & $\begin{array}{l}\text { Depois dos açúcares, os ácidos são os sólidos solúveis } \\
\text { presentes em maior quantidade no suco. A acidez é } \\
\text { determinada por titulação }\end{array}$ \\
\hline Ratio & $\begin{array}{l}\text { É a relação brix/acidez e fornece o grau de maturação } \\
\text { e qualidade do suco }\end{array}$ \\
\hline Variedade & $\begin{array}{l}\text { As variedades de laranja pêra, natal e valência são as } \\
\text { mais indicadas para a industrialização, enquanto a } \\
\text { variedade hamlin, por ser mais precoce, permite que } \\
\text { mas fornece um suco de qualidade inferior e de pouca } \\
\text { aceitação no mercado }\end{array}$ \\
\hline
\end{tabular}

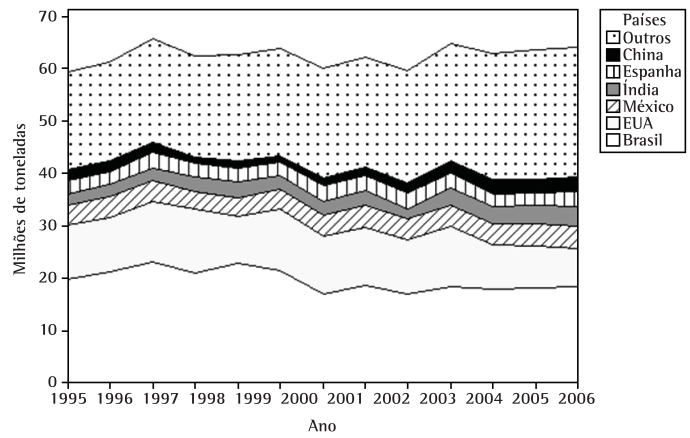

Figura 1. Produção de laranjas em milhares de toneladas. Fonte: FAO (FOOD..., 2009). de diferentes variedades, chamados de bases de suco) e produtos finais (sucos concentrados congelados de laranja obtidos pela mistura de diferentes bases) é particularmente importante, devido à combinação da sazonalidade da fruta e à relativa estabilidade na demanda por produtos. A utilização de modelos de programação linear para representar tal processo pode resultar em um poderoso instrumento para análise de decisões táticas e operacionais, conforme observado, por exemplo, em Munhoz (2000, 2009).

Entretanto, modelos de programação linear em geral admitem que os dados sejam precisamente conhecidos e iguais a determinados valores nominais. Bertsimas e Sim (2004) observam que a abordagem clássica de programação linear determinística não leva em consideração a influência de incertezas nos dados, na qualidade e factibilidade do modelo. A incorporação de incertezas a determinados parâmetros do modelo conduz a uma representação melhor da realidade, sendo a aplicação da abordagem de otimização robusta uma solução técnica e computacional atrativa por manter o modelo como um problema de programação linear, porém robusto. Essa abordagem é basicamente uma técnica de pior caso, que pretende obter soluções que minimizem o desvio máximo das variáveis aleatórias consideradas, de forma que seja assegurada a factibilidade do problema para o pior cenário das incertezas, sem grande perda de otimalidade e sem ter que considerar as distribuições de probabilidade associadas (BERTSIMAS; SIM, 2003; BERTSIMAS; THIELE, 2006). A técnica agrega mais flexibilidade no controle do conservadorismo adotado na análise da incerteza.

Dentro desse contexto, o presente trabalho apresenta uma abordagem de otimização robusta para dar tratamento às informações, visando suprir subsídios ao tomador de decisão na busca da excelência na gestão da cadeia de suprimentos da indústria cítrica. 0 planejamento agregado de produção é modelado com considerações de incertezas em alguns parâmetros,

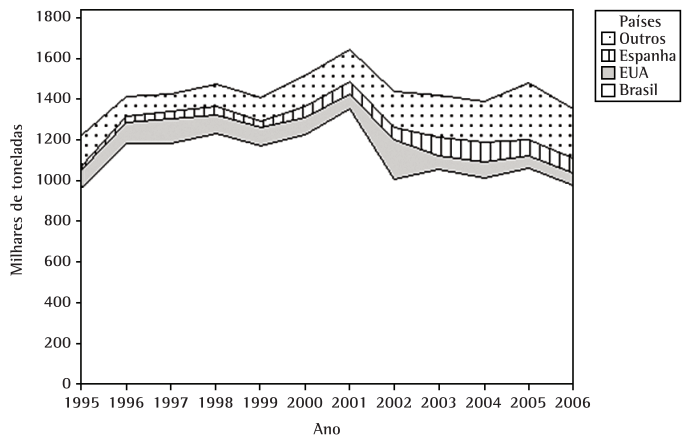

Figura 2. Exportação de suco concentrado de laranja em milhares de toneladas. Fonte: FAO (FOOD..., 2009). 
de modo a se construir uma ferramenta de suporte e análise efetiva à tomada de decisão em nível tático. A análise conjunta do processo de fornecimento das frutas até a geração dos produtos acabados para suprir a demanda é um aspecto importante deste trabalho. Utiliza-se como referência uma empresa típica do setor de produção de suco de laranja de distribuição internacional. No entanto, os conceitos aqui abordados são abrangentes o suficiente para serem aplicados a outras empresas similares do setor.

É fato que na prática do planejamento agregado dessa empresa tem-se significativo nível de incertezas em vários parâmetros do problema, entre eles podem-se citar: o ratio do suco (Quadro 1), a porcentagem de determinados componentes que é possivel de se utilizar no processo de mistura, o rendimento industrial (quantidade de laranja necessária para gerar uma tonelada de suco), a demanda esperada do suco, a disponibilidade de laranja, entre outros. 0 ratio da laranja é tido como um dos principais impactantes nesse planejamento. No setor há grande esforço depositado na tentativa de se precisar a curva que representa a evolução da maturação da fruta ao longo do tempo, aqui representada pelo ratio da base de suco de laranja. A principal linha utilizada pelas empresas é baseada em métodos estatísticos, que se resumem na análise de amostras de frutas, técnicas de regressão, combinadas com dados históricos e análise de especialistas sobre fatores diversos que impactam na maturação (por exemplo, clima, solo, trato de pomares, entre outros). Essa análise gera diferentes curvas, representando a evolução do ratio da laranja ao longo do tempo. Devido ao grau de incerteza associado a esse fato, é proposta deste trabalho uma abordagem de otimização robusta para tratar o problema em questão.

Em pesquisa bibliográfica identificaram-se poucos trabalhos que trataram do tema otimização aplicada à gestão da produção e operações na indústria cítrica. Por exemplo, Pinto (1996) utilizou programação linear inteira mista para apoiar decisões sobre sistemas de distribuição de suco concentrado de laranja. Caixeta Filho $(1993,2006)$ apresentou modelagens matemáticas para o gerenciamento da programação de colheita da laranja. Munhoz e Morabito (2001a, b, 2010) propuseram modelos determinísticos de programação linear e programação de metas para apoiar decisões no planejamento da produção e distribuição de suco de laranja. Os autores do presente trabalho desconhecem outros estudos na literatura explorando otimização determinística ou robusta no planejamento agregado de produção ou distribuição na indústria cítrica.

Este artigo está organizado da seguinte maneira: a seção 2 apresenta resumidamente o processo de produção do suco de laranja e o modelo determinístico de planejamento agregado de programação linear proposto em Munhoz e Morabito (2010). A seção 3 traz a abordagem de otimização robusta baseada nesse modelo, admitindo incertezas na matriz de coeficientes tecnológicos, mais precisamente nos parâmetros de acidez da base de suco que fazem parte das restrições de mistura do modelo. A seção 4 analisa os resultados obtidos da aplicação da abordagem com incertezas na acidez da base de suco, utilizando a linguagem de modelagem GAMS com o solver CPLEX (BROOKE; KENDRICK; MEERAUS, 1992) e os dados do estudo de caso realizado em Munhoz (2009). Avaliam-se o comportamento da abordagem e os resultados obtidos diante de diferentes níveis de proteção contra a violação das restrições de mistura. Finalmente, a seção 5 apresenta as conclusões deste trabalho e as perspectivas para trabalhos futuros.

\section{Processo de produção do suco de laranja e modelo determinístico de planejamento agregado}

A seguir é descrito resumidamente o processo e planejamento de produção do suco de laranja. Essa descrição é baseada em Munhoz (2009), onde o leitor poderá encontrar uma discussão mais detalhada desses tópicos.

\subsection{Processo de produção de suco}

A laranja é transportada até a fábrica por meio de caminhões que, ao chegarem à empresa, são descarregados através de rampas com acionamento hidráulico. A fruta é transportada por meio de correias e elevadores de canecas até os silos de estocagem de laranja. A fruta dos silos é retirada por meio de correias transportadoras e elevadores de canecas e encaminhada ao setor de lavagem, seleção e classificação e, em seguida, é processada nas extratoras, gerando as seguintes fases: suco com polpa; emulsão água com óleo e fragmentos de casca da laranja; e bagaço da laranja.

A fase suco com polpa é transportada por gravidade ao setor de filtragem e centrifugação. Nesse setor, são retirados do suco o excesso de polpa e outros defeitos que possam estar nele incorporados, além de se efetuar o ajuste do teor de polpa do produto dentro dos padrões desejados. 0 processo de industrialização segue pela concentração do suco, até a especificação desejada em evaporadores de múltiplos efeitos com filme descendente. Após concentrado, resfria-se o suco e armazena-se este a granel em câmaras frigoríficas. Nesse momento, tem-se o suco na forma de bases, 
que pode se entender como sendo um primeiro estágio de um processo de planejamento da produção. Em um segundo estágio tem-se o processo de mistura das diversas bases de suco, de forma a obterem-se os produtos finais.

As bases de suco, que estão armazenadas em câmaras frigoríficas com tanques em aço inoxidável, são bombeadas para tanques localizados no setor denominado blender em diferentes quantidades, de forma tal que se obtenha um produto dentro das especificações predeterminadas. Estando o produto conforme, este é bombeado novamente para as câmaras frigoríficas, para posterior embarque a granel, ou envasado em tambores metálicos de $200 \mathrm{~L}$, para posterior comercialização. 0 cálculo da quantidade de cada base a ser utilizada na mistura para resultar um ratio (brix/acidez) preestabelecido, é feito conforme a seguinte equação linear:

$$
\begin{aligned}
& A Q=A_{1} Q_{1}+A_{2} Q_{2}+\ldots+A_{n} Q_{n} \\
& \operatorname{com} Q=Q_{1}+Q_{2}+\ldots+Q_{n} \\
& R=\frac{B}{A}
\end{aligned}
$$

em que $R$ é o ratio final desejado para o produto acabado; $A$ é a acidez final desejada para o produto acabado; $Q$ é a quantidade desejada de produto acabado; $A_{i}$ é a acidez da base de suco $i(i=1,2, \ldots, n)$; $Q_{i}$ é a quantidade de base de suco $i(i=1,2, \ldots, n)$; e $B$ é o brix final médio do produto acabado, que no caso do problema é 66 , ou seja, $66 \%$ do produto são compostos por sólidos solúveis (vide definição de brix no Quadro 1).

Para algumas bases, além do cálculo acima, especificam-se também as quantidades limites (em porcentagem) de determinadas bases a serem adicionadas ao produto final. Nesse contexto, o planejamento utilizado para este trabalho se dá no nível tático da organização, quando se analisam simultaneamente os estoques de passagem de uma safra para outra, um plano de demanda por produtos acabados e um plano de disponibilidade de matéria-prima. A capacidade dos recursos produtivos é uma restrição particularmente importante.

0 plano de disponibilidade de laranja, que também pode ser chamado de planejamento de colheita, é elaborado a partir de diversos parâmetros, com destaque para a curva que representa a evolução da maturação da fruta ao longo do tempo, aqui representada pelo parâmetro ratio.

\subsection{Modelo determinístico de planejamento agregado}

Conforme mencionado, o ponto de partida para a abordagem de otimização robusta aqui proposta é o modelo de programação linear em Munhoz e Morabito (2010). Esse modelo incorpora o planejamento de colheita ao problema e também utiliza equações de mistura com base na acidez da laranja. 0 procedimento de cálculo da necessidade de matéria-prima (laranja) ao longo do horizonte de planejamento (uma safra) para atender a demanda dos produtos acabados, alinhada a uma política de cobertura de estoques para esses itens finais, é o cerne da modelagem. Como forma de viabilizar a geração do produto final, frente a limitações relacionadas à sazonalidade de disponibilidade da matéria-prima ao longo do horizonte de planejamento, é utilizado o procedimento de mistura de diversas bases de suco, ou seja, efetua-se o planejamento da produção em dois estágios: no primeiro estágio ocorre o processamento da laranja gerando produtos intermediários (bases de suco) e, no segundo estágio, efetua-se a mistura das diversas bases de suco de forma a se obterem os produtos finais.

0 modelo de programação linear (BAZARAA; JARVIS; SHERALI, 1990) obtido utiliza conceitos conhecidos da literatura de problemas de mistura e planejamento de produção com múltiplos produtos, estágios e períodos, conforme, por exemplo, em Johnson e Montgomery (1974), Williams (1978), Shapiro (1993), Al-Shammari e Dawood (1997), Arenales et al. (2007) e Nahmias (2009). 0 objetivo do modelo é minimizar o custo total com matéria-prima, estoques e falta de produto ao longo do horizonte de planejamento. As principais restrições para esse processo dizem respeito às especificações de qualidade da mistura, capacidade da produção, balanceamento de material e período de disponibilidade de matéria-prima.

0 modelo também utiliza conceitos de planejamento agregado e hierárquico (HAX; MEAL, 1975). A relevância da agregação de produtos para se obter modelos efetivos é reconhecida por diversos pesquisadores há algum tempo, como, por exemplo, em Geoffrion (1977), Zipkin (1982), Axsater e Jonsson (1984) e Shapiro (2001). A aplicação de conceitos de planejamento agregado está relacionada ao agrupamento dos diversos produtos demandados pelo mercado em famílias e, por conveniência, também se agrega a capacidade de processamento de produção como um todo, e não por unidade produtiva. Isso é interessante para empresas como a do presente estudo, com diversas unidades produtivas. A aplicação de conceitos de planejamento hierárquico se dá no aspecto de que o processo de planejamento tático fica subordinado ao planejamento estratégico do negócio.

Os Quadros 2-5 apresentam resumidamente a notação matemática utilizada pelo modelo em Munhoz e Morabito (2010) - a função objetivo e as restrições do modelo estão apresentadas resumidamente no Anexo 1. Essa notação e as Equações (A1)-(A17) do 
modelo são utilizadas pela abordagem de otimização robusta na próxima seção (para mais detalhes da notação e das equações do modelo, o leitor pode consultar a fonte).

\section{Abordagem por otimização robusta}

Conforme mencionado, é fato que na prática do planejamento agregado na indústria cítrica, tem-se significativo nível de incertezas em vários parâmetros do modelo (A1)-(A17) mencionado na seção 2.2 e apresentado resumidamente no Anexo 1. Entre eles, pode-se citar: o ratio da base $\left(\right.$ Ratio $\left._{j}\right)$, o ratio esperado da precoce (RatioH), a porcentagem esperada de precoce possível de se adicionar na base $j$ no

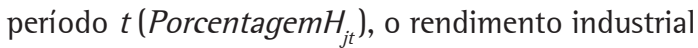
esperado da laranja $j$ gerando base $j$ no período $t$ em caixas de laranja por tonelada (Rendimento $B_{j t}$ ), a demanda esperada do suco $i$ no período $t$ em toneladas $\left(\right.$ Demand $_{i t}$ ), a disponibilidade de laranja, que nesse modelo é representada como estoque inicial de laranja base $\left(E L I_{f g o}\right)$ e laranja precoce $\left(E L H l_{f o}\right)$, entre outros.

Quadro 2. Índices utilizados no modelo determinístico.

\begin{tabular}{cl|}
\hline$i$ & Família de suco, que é referenciada apenas como suco \\
$t$ & Período de planejamento (mês) \\
$j$ & $\begin{array}{l}\text { Família de laranja que gera uma base de suco, que será } \\
\text { referenciada apenas como laranja e base, respectivamente }\end{array}$ \\
& $\begin{array}{l}\text { Fornecedor de laranja; este indice incorpora ao modelo a } \\
\text { obtenção de laranjas a partir de fontes diversas, como, por }\end{array}$ \\
$f$ & $\begin{array}{l}\text { exemplo, fruta própria, fruta adquirida através de contratos } \\
\text { de longo prazo, fruta adquirida no mercado spot, entre outras } \\
\text { fontes de fornecimento }\end{array}$ \\
$g$ & $\begin{array}{l}\text { Tipo de laranja; esse índice diferencia laranjas que apresentam } \\
\text { diferentes curvas de maturação ao longo de uma safra }\end{array}$ \\
\hline
\end{tabular}

\subsection{Incertezas na acidez das bases de suco}

0 ratio da base $\left(\operatorname{Ratio}_{j}\right)$ é tido como um dos principais impactantes nesse processo de planejamento. Com base nisso, propõe-se modelar o problema de programação linear determinístico (A1)-(A17) do Anexo 1 como um problema de programação linear robusto com incertezas na acidez da base de suco. Segue-se a abordagem elaborada por Bertsimas e Sim (2004), em que se admitem incertezas nos elementos da matriz tecnológica do modelo.

Seja o ratio da base $j\left(\operatorname{Ratio}_{j}\right)$, que é função da acidez da base $j\left(A B_{j}\right)$. Seja $K$ representando o conjunto de coeficientes $A B_{j}, j \in K$, que estão sujeitos a incerteza no modelo de otimização robusta. Cada entrada $A B_{j}$, $j \in K$, no modelo (A1)-(A17), é representada como uma variável aleatória $A \tilde{B}_{j}, j \in K$, com distribuição de probabilidade desconhecida, porém simétrica no intervalo $\left[A B_{j}-\hat{d}_{j}, A B_{j}+\hat{d}_{j}\right]$. 0 parâmetro $\hat{d}_{j}$ corresponde ao desvio da acidez da base $j$, isto é, representa o máximo desvio possível da acidez da base $j$ de seu valor médio $\left(A B_{j}\right)$.

Para as restrições de mistura (A7) e (A8) do modelo do Anexo 1 para obtenção dos produtos finais, conforme especificações a partir de bases e precoces, é introduzido o parâmetro $\Gamma$ com a função de ajustar a robustez do modelo contra o nível de conservadorismo da solução. Esse parâmetro conta a quantidade de parâmetros $A B_{j}$ (acidez da base $j$ ), $j \in K$, com incerteza, mas não é necessariamente inteiro, podendo assumir valores reais no intervalo $[0,|K|]$. A modelagem aplicada a seguir tem a propriedade, segundo Bertsimas e Sim (2004), de que

Quadro 3. Dados utilizados no modelo determinístico.

\begin{tabular}{|c|c|}
\hline$E L l_{f g o}$ & Estoque inicial $(t=0)$ de laranja base no fornecedor $f$ do tipo $g$ em caixas de laranja de $40,8 \mathrm{~kg}$ \\
\hline$E L H l_{f o}$ & Estoque inicial $(t=0)$ de laranja precoce no fornecedor $f$ em caixas de laranja de $40,8 \mathrm{~kg}$ \\
\hline$H S I_{i 0}$ & Estoque inicial $(t=0)$ de suco $i$ em toneladas \\
\hline$H H l_{o}$ & Estoque inicial $(t=0)$ de precoce $i$ em toneladas \\
\hline$H B l_{j 0}$ & Estoque inicial ( $t=0$ ) de base $j$ em toneladas \\
\hline $\mathrm{FSI}_{i 0}$ & Falta de estoque inicial $(t=0)$ de suco $i$ em toneladas \\
\hline$F H l_{o}$ & Falta de estoque inicial $(t=0)$ de precoce $i$ em toneladas \\
\hline$F B l_{j 0}$ & Falta de estoque inicial $(t=0)$ de base $j$ em toneladas \\
\hline$D_{t}$ & Máximo de dias disponíveis para processamento no período $t$ \\
\hline$D T L_{t}$ & Mínimo de dias disponíveis para processamento no período $t$ \\
\hline$C P_{t}$ & Capacidade de processamento diária no período $t$ em caixas de laranja de $40,8 \mathrm{~kg}$ \\
\hline RatioSH $_{i}$ & Ratio máximo do suco $i$ \\
\hline RatioSL $_{i}$ & Ratio mínimo do suco $i$ \\
\hline Ratio $_{j}$ & Ratio da base $j$ \\
\hline RatioH & Ratio esperado da precoce \\
\hline PorcentagemH $H_{j t}$ & Porcentagem esperada de precoce possível de se adicionar na base $j$ no período $t$ \\
\hline Rendimento $B_{j t}$ & Rendimento industrial esperado da laranja $j$ gerando a base $j$ no período $t$ em caixas de laranja/tonelada \\
\hline RendimentoH & $\begin{array}{l}\text { Rendimento industrial esperado da laranja precoce gerando base precoce no período } t \text { em caixas de } \\
\text { laranja/tonelada }\end{array}$ \\
\hline Demanda $_{i t}$ & Demanda esperada do suco $i$ no período $t$ em toneladas \\
\hline
\end{tabular}


Quadro 4. Outros dados utilizados no modelo determinístico.

\begin{tabular}{|c|c|}
\hline CustoHS ${ }_{i t}$ & $\begin{array}{l}\text { Custo de armazenagem do suco } i \text { no período } t \\
\text { (unidades monetárias/tonelada) }\end{array}$ \\
\hline CustoHH $_{t}$ & $\begin{array}{l}\text { Custo de armazenagem de precoce no período } t \\
\text { (unidades monetárias/tonelada) }\end{array}$ \\
\hline CustoHB ${ }_{J T}$ & $\begin{array}{l}\text { Custo de armazenagem de base } j \text { no período } t \\
\text { (unidades monetárias/tonelada) }\end{array}$ \\
\hline CustoFS $_{i t}$ & $\begin{array}{l}\text { Custo de falta do suco } i \text { no período } t \text { (unidades } \\
\text { monetárias/tonelada) }\end{array}$ \\
\hline CustoFH $_{t}$ & $\begin{array}{l}\text { Custo de falta de precoce no período } t \text { (unidades } \\
\text { monetárias/tonelada) }\end{array}$ \\
\hline CustoFB $_{J T}$ & $\begin{array}{l}\text { Custo de falta da base } j \text { no período } t \text { (unidades } \\
\text { monetárias/tonelada) }\end{array}$ \\
\hline CustoQL $_{\text {fgt }}$ & $\begin{array}{l}\text { Custo da laranja base do fornecedor } f \text { do tipo } g \text { no } \\
\text { período } t \text { (unidades monetárias/caixa de } 40,8 \mathrm{~kg} \text { ) }\end{array}$ \\
\hline CustoQLH $_{f t}$ & $\begin{array}{l}\text { Custo da laranja precoce do fornecedor } f \text { no } \\
\text { período } t \text { (unidades monetárias/caixa de } 40,8 \mathrm{~kg} \text { ) }\end{array}$ \\
\hline$D L_{\text {fgit }}$ & $\begin{array}{l}\text { Indica se tem disponibilidade da laranja base } j \text { do } \\
\text { fornecedor } f \text { do tipo } g \text { no período } t(0=\text { não tem; } \\
1=\text { tem) }\end{array}$ \\
\hline$D H_{f t}$ & $\begin{array}{l}\text { Indica se tem disponibilidade da laranja precoce do } \\
\text { fornecedor } f \text { no período } t(0=\text { não tem; } 1=\text { tem })\end{array}$ \\
\hline$C F_{f t}$ & $\begin{array}{l}\text { Capacidade de fornecimento de laranja (base e } \\
\text { precoce) do fornecedor } f \text { no período } t \text { em caixas de } \\
\text { laranja de } 40,8 \mathrm{~kg}\end{array}$ \\
\hline$C P H_{t}$ & $\begin{array}{l}\text { Capacidade de processamento industrial máxima no } \\
\text { período } t \text { em caixas de laranja de } 40,8 \mathrm{~kg}\end{array}$ \\
\hline$C P L_{t}$ & $\begin{array}{l}\text { Capacidade de processamento industrial mínima no } \\
\text { período } t \text { em caixas de laranja de } 40,8 \mathrm{~kg}\end{array}$ \\
\hline $\mathrm{ASH}_{i}$ & Acidez máxima do suco $i$ \\
\hline$A S L_{i}$ & Acidez mínima do suco $i$ \\
\hline$A B_{j}$ & Acidez da base $j$ \\
\hline$A H$ & Acidez da precoce \\
\hline
\end{tabular}

se na realização desse experimento aleatório (i.e., com as variáveis aleatórias $\left.A \tilde{B}_{j}, j \in K\right)$ variar até $\lfloor\Gamma\rfloor$ coeficientes $A B_{j}, j \in K$, e um coeficiente $A B_{j_{t}}, j_{t} \in K$, variando da parte fracionária $(\Gamma-\lfloor\Gamma\rfloor) \hat{d}_{j t}$, então a solução do modelo de otimização robusta será factível deterministicamente nesse experimento aleatório. Além disso, mesmo se mais do que $\lfloor\Gamma\rfloor$ coeficientes variarem, então essa solução robusta será factível nesse experimento aleatório com alta probabilidade.

Para se construir o modelo de otimização robusta a partir do modelo determinístico (A1)-(A17) (vide Anexo 1), é necessário modificar a formulação das restrições de mistura (A7) e (A8), de forma a considerar incerteza nos parâmetros $A B_{j}$, conforme a seguir:

$$
\begin{aligned}
& {\left[\sum_{j}\left(Q B_{j t i} \times A B_{j}+Q H_{j t i} \times A H\right)\right]-\left(\sum_{j}\left(Q B_{j t i}+Q H_{j t i}\right)\right) \times A S H_{i}+} \\
& \max _{\Theta}\left\{\sum_{j \in S} \hat{d}_{j} y_{j t i}+(\Gamma-\lfloor\Gamma\rfloor) \hat{d}_{j t} y_{j t}\right\} \leq 0 ; \forall i, t \\
& {\left[\sum_{j}\left(Q B_{j t i} \times A B_{j}+Q H_{j t i} \times A H\right)\right]-\left(\sum_{j}\left(Q B_{j t i}+Q H_{j t i}\right)\right) \times A S L_{i}-} \\
& \max _{\Theta}\left\{\sum_{j \in S} \hat{d}_{j} y_{j t i}+(\Gamma-\lfloor\Gamma\rfloor) \hat{d}_{j_{t}} y_{j t t i}\right\} \geq 0 ; \forall i, t
\end{aligned}
$$

\begin{tabular}{|c|c|}
\hline$Q L_{\text {fojt }}$ & $\begin{array}{l}\text { Quantidade de laranja } j \text { gerando base } j \text { a ser } \\
\text { processada no período } t \text { proveniente do fornecedor } \\
f \text { do tipo } g \text {, em caixas de } 40,8 \mathrm{~kg}\end{array}$ \\
\hline$Q L H_{f i}$ & $\begin{array}{l}\text { Quantidade de laranja precoce gerando base } \\
\text { precoce no periodo } t \text { proveniente do fornecedor } f \text {, } \\
\text { em caixas de } 40,8 \mathrm{~kg}\end{array}$ \\
\hline$Q S_{i t}$ & $\begin{array}{l}\text { Quantidade de suco } i \text { produzido no período } t \text { em } \\
\text { toneladas }\end{array}$ \\
\hline$Q H P_{t}$ & $\begin{array}{l}\text { Quantidade de precoce produzida no período } t \mathrm{em} \\
\text { toneladas }\end{array}$ \\
\hline$Q B P_{J T}$ & $\begin{array}{l}\text { Quantidade de base } j \text { produzida no periodo } t \text { em } \\
\text { toneladas }\end{array}$ \\
\hline$Q H_{j t i}$ & $\begin{array}{l}\text { Quantidade de precoce consumida na base } j \text { para } \\
\text { produzir suco } i \text { no período } t \text { em toneladas }\end{array}$ \\
\hline$Q B_{j t i}$ & $\begin{array}{l}\text { Quantidade de base } j \text { consumida para produzir suco } \\
i \text { no período } t \text { em toneladas }\end{array}$ \\
\hline$H S_{i t}$ & Estoque de suco $i$ no período $t$ em toneladas \\
\hline$H H_{t}$ & Estoque de precoce no período $t$ em toneladas \\
\hline$H B_{J T}$ & Estoque de base $j$ no período $t$ em toneladas \\
\hline$F S_{i t}$ & Falta de suco $i$ no período $t$ em toneladas \\
\hline$F H_{t}$ & Falta de precoce no período $t$ em toneladas \\
\hline$F B_{J T}$ & Falta de base $j$ no período $t$ em toneladas \\
\hline$E L_{f g t}$ & $\begin{array}{l}\text { Estoque de laranja base no fornecedor } f \text { do tipo } g \\
\text { no período } t \text { em caixas de laranja de } 40,8 \mathrm{~kg}\end{array}$ \\
\hline$E L H_{f i}$ & $\begin{array}{l}\text { Estoque de laranja precoce no fornecedor } f \text { no } \\
\text { período } t \text { em caixas de laranja de } 40,8 \mathrm{~kg}\end{array}$ \\
\hline
\end{tabular}

Quadro 5. Variáveis de decisão utilizadas no modelo determinístico.

$$
-y_{j t i} \leq Q B_{j t i} \leq y_{j t i} ; \forall j, t, i
$$

$y_{j t i} \geq 0 ; \forall j, t, i$

onde $\Theta=\{S \cup\{t\}|S \subseteq K| S \mid,=\lfloor\Gamma\rfloor, t \in K / S\}, y_{j t t}$ é uma variável de modelagem da incerteza simétrica. Essa variável está associada à variável de decisão $Q B_{j t i}$ (Quadro 5) e ao parâmetro $\hat{d}_{j}$ (chamado de espaço de incerteza). Para mais detalhes das duas últimas expressões acima $\left(-y_{j t i} \leq Q B_{j t i} \leq y_{j t i} ; y_{j t i} \geq 0\right)$, o leitor pode consultar Soyster (1973). Pode ser mostrado que, na otimalidade do modelo de otimização robusta, tem-se que $y_{j t i}=\left|Q B_{j t i}\right|, \forall j, t, i$; como no presente trabalho tem-se que $Q B_{j t i} \geq 0$, então, na otimalidade, $y_{j t i}=Q B_{j t i}$ $\forall j, t, i$ (vide BERTSIMAS; SIM, 2004). 0 último termo do lado esquerdo das restrições (1) e (2) é chamado função de proteção dessas restrições:

$$
\beta_{t i}\left(y^{*}, \Gamma\right)=\max _{\Theta}\left\{\sum_{j \in S} \hat{d}_{j} y_{j t i}^{*}+(\Gamma-\lfloor\Gamma\rfloor) \hat{d}_{j_{t}} y_{j t i}^{*}\right\}
$$

onde $\Theta=\{S \cup\{t\}|S \subseteq K| S \mid,=\lfloor\Gamma\rfloor, t \in K / S\}, y^{*}$ denota um vetor $y$ factível para o problema. Pode ser mostrado que o valor dessa função de proteção das restrições de mistura é igual ao valor da função objetivo do seguinte problema de otimização linear (BERTSIMAS; SIM, 2004):

maximizar $\sum_{j \in K} \hat{d}_{j} y_{j t i}^{*} z_{j}$ 
sujeito a:

$$
\begin{aligned}
& \sum_{j \in K} z_{j} \leq \Gamma \\
& 0 \leq z_{j} \leq 1, \forall j \in K
\end{aligned}
$$

Seguindo o mesmo raciocínio de Bertsimas e Sim (2004), o dual do problema (4)-(6) é dado por:

minimizar $\Gamma z+\sum_{j \in K} p_{j}$

sujeito a:

$$
z+p_{j} \geq \hat{d}_{j} y_{j t i}^{*} ; \forall j \in K, t, i
$$

$$
Z \geq 0
$$

$$
p_{j} \geq 0 ; \forall j \in K
$$

em que $z$ e $p_{j}$ são as variáveis duais associadas às restrições (5) e (6), respectivamente; $z$ é chamada variável de robustez e $p_{j}$ é uma variável auxiliar robusta. Por dualidade forte, uma vez que o problema (4)-(6) é factivel e limitado por todo $\Gamma \in[0,|K|]$, então o problema dual (7)-(10) também é factível e limitado, e os valores de duas funções objetivo coincidem. Dessa forma, tem-se que $\beta_{t i}\left(y^{*}, \Gamma\right)$ é igual ao valor da função objetivo do problema (7)-(10).

Substituindo o problema (7)-(10) nas restrições (1) e (2), obtêm-se o modelo de otimização linear robusto definido a seguir. Por motivo de economia de espaço, é apresentado apenas o que é novo ou muda em relação ao material da seção 2.2 e o modelo determinístico (A1)-(A17) do Anexo 1.

\subsection{Modelo de programação linear robusto}

\subsubsection{Indices}

Mantêm-se os mesmos índices definidos no Quadro 2 da seção 2.2 e acrescenta-se o seguinte indice:

$k(j)=$ família de laranja $j$ que gera a base de suco $j$ com incerteza na acidez, onde $j \in K$;

0 objetivo desse índice é controlar os parâmetros da família de suco $j$ que apresentam incertezas na acidez.

\subsubsection{Dados}

Mantêm-se os mesmos dados definidos nos Quadros 3 e 4 da seção 2.2 e acrescentam-se os parâmetros $\Gamma$ (parâmetro para ajuste da robustez do modelo) e $\hat{d}_{j}$ (desvio da acidez da base $j$ ), definidos na seção 3.1 .

\subsubsection{Variáveis de decisão}

Mantêm-se as mesmas variáveis de decisão definidas na Quadro 5 da seção 2.2 e acrescentam-se as variáveis $z$ (variável de robustez), $p_{j}$ (variável auxiliar robusta) e $y_{j t i}$ (variável de modelagem da incerteza simétrica), definidas na seção 3.1.

\subsubsection{Função objetivo}

A função objetivo se mantém a mesma apresentada na Equação (A1) do Anexo 1.

\subsubsection{Restrições}

A restrição de o nível total de processamento desse planejamento tático respeitar (planejamento hierárquico) a definição estratégica de nível de processamento, se mantém igual à restrição (A2) do Anexo 1. A restrição de níveis máximo e mínimo de processamento industrial é igual às restrições (A3) do Anexo 1. As equações de balanceamento de material são iguais às Equações (A4), (A5) e (A6) do Anexo 1.

Equações de mistura para obtenção dos produtos finais conforme especificações a partir de bases e precoces:

$$
\begin{aligned}
& {\left[\sum_{j}\left(Q B_{j t i} \times A B_{j}+Q H_{j t i} \times A H\right)\right]+\Gamma z-} \\
& \left(\sum_{j}\left(Q B_{j t i}+Q H_{j t i}\right)\right) \times A S H_{i}+\sum_{j \in K} p_{j} \leq 0 ; \forall i, t
\end{aligned}
$$

$$
\begin{aligned}
& {\left[\sum_{j}\left(Q B_{j t i} \times A B_{j}+Q H_{j t i} \times A H\right)\right]-\Gamma z-} \\
& \left(\sum_{j}\left(Q B_{j t i}+Q H_{j t i}\right)\right) \times A S L_{i}+\sum_{j \in K} p_{j} \geq 0 ; \forall i, t \\
& Q B_{j t i} \leq y_{j t i} ; \forall j, t, i \\
& Z=p_{j} \geq \hat{d}_{j} y_{j t i} ; \forall j \in K, t, i \\
& Z \geq 0
\end{aligned}
$$

$p_{j} \geq 0 ; \forall j \in K$

$y_{j t i} \geq 0 ; \forall j, t, i$ 
$Q H_{j t i} \leq$ Porcentagem $_{j t} \times\left(Q B_{j t i}+Q H_{j t i}\right) ; \forall j, t, i$

$Q S_{i t}=\sum_{j}\left(Q B_{j t i}+Q H_{j t i}\right) ; \forall i, t$

As Equações (11) a (17) são as equações de mistura admitindo-se incertezas na acidez da base $j\left(A B_{j}\right)$, onde o desenvolvimento para se obter tal formulação é o descrito na seção 3.1. As Equações (18) e (19) são idênticas às Equações (A9) e (A10), respectivamente, do Anexo 1. As equações de relação de quantidade de laranja gerando quantidades de bases e precoces, incorporando o fator disponibilidade de fruta nos cálculos, são iguais às Equações (A11) a (A14) do Anexo 1. As equações de balanceamento de material para fornecimento de laranja são iguais às Equações (A15) e (A16) do Anexo 1. As restrições de capacidade de fornecimento de laranja são iguais às Equações (A17) do Anexo 1. Finalmente, tem-se que todas as variáveis de decisão para esse modelo são não negativas, assim como no modelo do Anexo 1.

Portanto, o modelo de programação linear robusto é composto pela função objetivo $(\mathrm{A} 1)$ e as restrições (A2) a (A6) e (A11) a (A17) do Anexo 1, mais as restrições (11) a (19) (que substituem as restrições (A7), (A8), (A9) e (A10) do Anexo 1). A solução desse modelo é chamada de solução robusta.

\subsection{Limitantes para probabilidade de violação das restrições}

Uma das vantagens da abordagem proposta por Bertsimas e Sim $(2003,2004)$ é a possibilidade de inferir a probabilidade de violação das restrições para os casos em que a variável aleatória ultrapasse o espaço de incerteza considerado. A demonstração da probabilidade de violação dos limites das restrições é apresentada em Sim (2004), onde se tem que o parâmetro $\Gamma$ faz o balanceamento (trade-off) entre a probabilidade de violação e o efeito para a função objetivo do problema nominal, denominado preço da robustez.

Bertsimas e Sim (2004) demonstram que, sob o modelo de dados incertos apresentado no início desta seção, a solução robusta é factível com alta probabilidade, e apresentam alguns limitantes para a probabilidade de violação da restrição. Na sequência apresentam-se dois desses limitantes, que são utilizados nesse trabalho na seção 4.

Limitante 1:

$$
\frac{1}{2^{n}}\left[(1-\mu)\left(\begin{array}{c}
n \\
\lfloor v\rfloor
\end{array}\right)+\sum_{r=\lfloor v\rfloor+1}^{n}\left(\begin{array}{l}
n \\
r
\end{array}\right)\right]
$$

com $n=|K|, v=(\Gamma+n) / 2$ e $\mu=v-\lfloor v\rfloor$.
Limitante 2:

$$
(1-\mu) C(n,\lfloor v\rfloor)+\sum_{r=\lfloor v\rfloor+1}^{n} C(n, r)
$$

com

$C(n, r)=\frac{1}{2^{n}}$, se $r=0$ ou $r=n$,

$C(n, r)=\frac{1}{\sqrt{2 \pi}} \sqrt{\frac{n}{(n-r) r}}$

$\exp \left[n \log \left(\frac{n}{2(n-r)}\right)+r \log \left(\frac{n-r}{r}\right)\right]$, caso contrário.

Enquanto o limitante 1 é o melhor possível (BERTSIMAS; SIM, 2004), ele apresenta dificuldades computacionais para avaliar a soma de funções de combinação para $n$ grande. Por essa razão os autores apresentam o limitante 2 , que é simples de ser calculado e também razoavelmente bom (ver demonstração em BERTSIMAS; SIM, 2004).

Os autores também ilustram a escolha de $\Gamma$ como uma função de $n$, de forma que a probabilidade de uma restrição do problema ser violada é menos de 1\%. A Tabela 1 apresenta o valor a ser utilizado para $\Gamma$, dependendo da quantidade de parâmetros sujeitos a incerteza $n$, de forma que os limitantes 1 e 2 apresentem probabilidade menor que 1\% de que uma restrição seja violada. Por exemplo, para $n=100$ parâmetros, deve-se escolher $\Gamma=24,3$; dessa forma os limitantes 1 e 2 oferecem probabilidade de violação da restrição menor que $1 \%$. Os autores descrevem que para restrições com poucos parâmetros com incerteza, é necessário assegurar quase que proteção total, o que é equivalente ao método de Soyster (1973). Porém, para restrições com vários parâmetros com incerteza, a abordagem proposta fornece soluções menos conservativas relativamente ao método de Soyster (ver Tabela 1).

\section{Resultados computacionais}

Nesta seção avalia-se o comportamento do modelo de otimização robusta da seção 3 para resolver o problema de planejamento do estudo de caso em Munhoz (2009) e Munhoz e Morabito (2010). Esse comportamento é estudado para diferentes níveis de proteção contra a violação da restrição de mistura, ou seja, variando-se $\Gamma=0,1,2, \ldots, 7$ (note que $|K|=7$ bases é o número máximo de parâmetros sujeitos a incerteza na modelagem atual). Convém notar que fazendo-se $\Gamma=0$, a abordagem de otimização robusta corresponde ao modelo determinístico (A1)-(A17) do Anexo 1. Por outro lado, $\Gamma=7$ indica que todos os coeficientes da restrição de mistura estão sujeitos a incertezas e, neste caso, a abordagem de otimização robusta torna-se equivalente à apresentada em Soyster 
(1973). Os valores intermediários para $\Gamma$ (entre 1 e 6$)$ representam a abordagem de Bertsimas e Sim (2004). Como os modelos envolvidos com cada valor de $\Gamma$ são de otimização linear, os tempos computacionais para resolvê-los pelo GAMS/CPLEX são bem aceitáveis, da ordem de poucos segundos no computador utilizado (com processador Intel(R) CPU T2300, $1.66 \mathrm{GHz}$ e $0.99 \mathrm{~GB}$ de memória RAM).

A Tabela 2 ilustra os limites para os parâmetros de acidez das bases de suco sujeitos a incertezas, e o respectivo impacto no ratio das bases de suco. Note que para o cenário em questão, admite-se um desvio na acidez da base de suco de $2 \%$. Quando no limite inferior de acidez, tem-se uma redução no valor do parâmetro de $2 \%$; a contrapartida no ratio da base é um incremento de aproximadamente $2 \%$ em relação ao valor médio (i.e., $\left.0,98 \approx(1,02)^{-1}\right)$. Da mesma forma, quando no limite superior de acidez, tem-se um acréscimo de 2\% em relação ao valor médio; a contrapartida no ratio da base é uma redução de aproximadamente $2 \%$ em relação ao valor médio.

\subsection{Trade-off entre custo total e probabilidade de violação das restrições (risco)}

Para avaliar o balanceamento entre a probabilidade de violação dos limites das restrições de mistura e o efeito para a função objetivo relativamente ao problema nominal, o qual Bertsimas e Sim (2004) denominam preço da robustez, utiliza-se o limitante descrito na seção 3.3. Aplicando-se esse limitante, obtiveram-se os resultados da Tabela 3

Tabela 1. Escolha de $\Gamma$ como função de $n=|K|$ de forma que a probabilidade de uma restrição ser violada seja menor que $1 \%$.

\begin{tabular}{cc}
\hline$n=|K|$ & $\Gamma$ para limitantes 1 e 2 \\
\hline 5 & 5 \\
10 & 8,2 \\
100 & 24,3 \\
200 & 33,9 \\
2000 & 105 \\
\hline
\end{tabular}

Fonte: Bertsimas e Sim (2004). e da Figura 3. Nota-se que para $\Gamma=0$, tem-se 0 modelo determinístico (A1)-(A17) do Anexo 1 sem prejuizo do valor ótimo da função objetivo, porém com uma probabilidade de violação da restrição de mistura elevada (64\%). Aumentando-se a proteção, ou seja, aumentando-se o valor de $\Gamma$ (admitindo-se que mais parâmetros das restrições de mistura estão sujeitos a incertezas), nota-se que a probabilidade de violação da restrição de mistura reduz. No entanto, o custo total da operação aumenta, o que vem a ser o preço da robustez discutido em Bertsimas e Sim (2004). E quando se aplica a proteção máxima $(\Gamma=7)$, tem-se o ponto de máximo prejuízo do valor ótimo da função objetivo em relação ao problema nominal, com penalização de $64,91 \%$, mas com probabilidade de violação das restrições de mistura nula.

Com base nesses resultados, constata-se que, embora seja desejável utilizar proteção total $(\Gamma=7)$, dessa forma anulando-se a probabilidade de violação das restrições, a penalidade associada é muito alta (aumento de 64,91\% do custo total), relativamente ao valor obtido no problema nominal determinístico. 0 nível de proteção vai depender da postura do gestor em optar por correr mais (ou menos) riscos, para incorrer em menos (ou mais) custos.

\subsection{Efeitos das soluções robustas nas quantidades produzidas}

Os resultados obtidos pela abordagem de otimização robusta variando-se o parâmetro $\Gamma$ implicam em variações nas quantidades de bases de suco produzidas e em tendências de aumento nas quantidades de suco geradas, conforme ilustrado na Tabela 4. Sendo que "BA11", "BA13" etc., na Tabela 4, são bases de suco com ratio 11 , ratio 12 , e assim sucessivamente. 0 aumento das quantidades de suco produzidas gera formação de estoques, o que está de acordo com o conceito de gestão da produção e inventário de que, na presença de mais incertezas, uma possível ação é aumentar os níveis de estoques de materiais.

Tabela 2. Intervalo de variação do parâmetro acidez da base de suco e o respectivo impacto no ratio da base de suco.

\begin{tabular}{|c|c|c|c|c|c|c|c|c|}
\hline \multicolumn{9}{|c|}{ Desvio da acidez da base e equivalência no ratio } \\
\hline & \multicolumn{5}{|c|}{ Acidez da base } & \multicolumn{3}{|c|}{ Ratio da base } \\
\hline & $A B_{j}-d_{j}$ & $A B_{j}$ & $A B_{j}+d_{j}$ & $-d_{j}(\%)$ & $d_{j}(\%)$ & Ratio $B_{j}-d_{j}$ & Ratio $_{j}$ & Ratio $_{j}+d_{j}$ \\
\hline BA11 & 5,624 & 5,739 & 5,854 & -2 & 2 & 11,7 & 11,5 & 11,3 \\
\hline BA13 & 4,791 & 4,889 & 4,987 & -2 & 2 & 13,8 & 13,5 & 13,2 \\
\hline BA14 & 4,461 & 4,552 & 4,643 & -2 & 2 & 14,8 & 14,5 & 14,2 \\
\hline BA15 & 4,173 & 4,258 & 4,343 & -2 & 2 & 15,8 & 15,5 & 15,2 \\
\hline BA16 & 3,920 & 4,000 & 4,080 & -2 & 2 & 16,8 & 16,5 & 16,2 \\
\hline BA17 & 3,696 & 3,771 & 3,847 & -2 & 2 & 17,9 & 17,5 & 17,2 \\
\hline BA18 & 3,496 & 3,568 & 3,639 & -2 & 2 & 18,9 & 18,5 & 18,1 \\
\hline
\end{tabular}


0 modelo de otimização robusta reage gerando diferentes planos de mistura, conforme se aumenta $\Gamma$. 0 ratio resultante desses planos de mistura, para cada produto ao longo do horizonte de planejamento, tende para um valor intermediário da especificação de ratio do produto acabado, de forma a proteger contra os desvios nos parâmetros com incertezas. Ou seja, o modelo reage produzindo diferentes planos de mistura para diferentes valores de $\Gamma$, de modo a gerar os produtos acabados, respeitando os limites da especificação de ratio e de consumo de base de suco precoce (máximo 15\% em cada processo de mistura). lsso é demonstrado, por exemplo, nas Tabelas 5 e 6 , cujas primeiras nove colunas correspondem à solução robusta obtida para $\Gamma=7$ considerando desvio de

Tabela 3. Resultados das soluções robustas utilizando limitante.

\begin{tabular}{cccc}
\hline$\Gamma$ & $\begin{array}{c}\text { Probabilidade } \\
\text { de violação da } \\
\text { restrição (\%) }\end{array}$ & Valor ótimo & Acréscimo (\%) \\
\hline 0 & 64 & 320304896 & - \\
1 & 50 & 346268681 & 8,11 \\
2 & 36 & 372403977 & 16,27 \\
3 & 23 & 409699968 & 27,91 \\
4 & 14 & 447806986 & 39,81 \\
5 & 6 & 479177451 & 49,60 \\
6 & 4 & 511860777 & 59,80 \\
7 & 0 & 528208770 & 64,91 \\
\hline
\end{tabular}

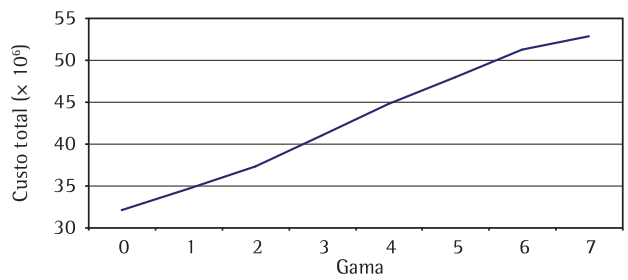

Figura 3. Custo total como função de $\Gamma$ (preço da robustez) utilizando limitante.
2\% na acidez de todas as bases, ou seja, trata-se da solução robusta de Soyster (1973).

Nota-se que o formato proposto nessas tabelas é prático para suportar a implementação desse plano de mistura. As tabelas são compostas por quadros, representando cada produto final: linhas representando os diferentes períodos de planejamento, e colunas indicando quanto de cada componente deve ser consumido em cada período de planejamento. As últimas três colunas de cada quadro indicam a acidez, o ratio e a porcentagem de precoce adicionada para o produto acabado resultante em cada período. No caso da Tabela 5, consideramos que o desvio na acidez de todas as bases será de 0\% (i.e., após a realização do experimento aleatório, os valores das variáveis aleatórias $A \tilde{B}_{j}$ observados coincidirão com os valores nominais dos coeficientes $A B_{j}, j \in K$ ), enquanto no caso da Tabela 6 , consideramos que o desvio na acidez de todas as bases será de $2 \%$ (i.e., os valores das variáveis aleatórias $A \tilde{B}_{j}$ observados serão os mais desfavoráveis possíveis). Por exemplo, note na Tabela 5 que para se produzir o produto acabado "PA13" no período (mês) 1, são necessárias $511 \mathrm{t}$ de "precoce", $1407 \mathrm{t}$ de base "BA11", $966 \mathrm{t}$ de "BA15" e 528 t de base "BA16". Considerando-se que o desvio na acidez das bases será de $0 \%$, essa mistura resultará em uma acidez 4,897, ratio 13,48 e uma adição de 15\% de "precoce", resultados esses que estarão dentro das especificações impostas pelo modelo.

Note nas últimas colunas da Tabela 5 que os valores de ratio do produto acabado serão muito próximos de seus valores médios da faixa de especificação. Em contrapartida, na Tabela 6, observa-se que a especificação de ratio de todos os produtos acabados ao longo do horizonte de planejamento continuarão respeitando os limites das respectivas especificações (três últimas colunas da tabela). Além disso, nota-se que a especificação de ratio do produto "PA14" para essa situação de pior caso estará no limite inferior da faixa permissível, ou seja, o limite inferior da

Tabela 4. Quantidade de bases de suco produzida em função da variação de $\Gamma$ (valores em toneladas de suco).

\begin{tabular}{|c|c|c|c|c|c|c|c|c|}
\hline & \multicolumn{8}{|c|}{ Quantidade de base produzida em função de gama (valores em toneladas de suco) } \\
\hline & \multicolumn{8}{|c|}{ Valores de $\Gamma$} \\
\hline & 0 & 1 & 2 & 3 & 4 & 5 & 6 & 7 \\
\hline BA11 & 33.311 & 38.279 & 41.387 & 46.221 & 51.082 & 60.668 & 69.660 & 79.500 \\
\hline BA13 & 6.552 & 5.017 & 8.669 & 10.902 & 16.886 & 16.294 & 16.013 & - \\
\hline BA14 & 40.644 & 27.954 & 28.219 & 21.084 & 19.980 & 21.612 & 17.626 & - \\
\hline BA15 & 26.260 & 39.054 & 30.180 & 26.978 & 23.641 & 20.420 & 31.831 & 34.762 \\
\hline BA16 & 17.572 & 22.207 & 29.330 & 27.753 & 22.362 & 18.529 & 19.219 & 39.075 \\
\hline BA17 & 29.336 & 23.575 & 23.281 & 21.440 & 24.946 & 21.306 & 20.184 & 26.157 \\
\hline BA18 & - & - & 2 & 6.912 & 9.491 & 15.391 & 14.031 & 6.626 \\
\hline Precoce & 26.325 & 23.914 & 23.791 & 25.210 & 25.869 & 26.410 & 27.742 & 27.742 \\
\hline Total & 180.000 & 180.000 & 184.859 & 186.500 & 194.257 & 200.630 & 216.306 & 213.862 \\
\hline
\end{tabular}


Tabela 5. Resultado do plano de mistura para $\Gamma=7$ gerado pelo modelo apresentado na seção 3 - as três últimas colunas consideram que o desvio na acidez da base será de $0 \%$.

\begin{tabular}{|c|c|c|c|c|c|c|c|c|c|c|c|c|}
\hline \multirow{3}{*}{ Mês } & & \multicolumn{8}{|c|}{ (consumo esperado de precoce: máximo de 15\%) } & \multirow[t]{2}{*}{ desvio: } & \multicolumn{2}{|l|}{$0 \%$} \\
\hline & & Precoce & BA11 & BA13 & BA14 & BA15 & BA16 & BA17 & BA18 & & & \\
\hline & & \multicolumn{8}{|c|}{ Quantidade em toneladas } & Acidez & Ratio & \%Precoce \\
\hline 1 a 12 & $\begin{array}{c}\text { PA11 } \\
\text { (de } 11,00 \text { a } 11,99 \text { ) }\end{array}$ & 0 & 2613 & 0 & 0 & 0 & 0 & 0 & 0 & 5,739 & 11,50 & 0,0 \\
\hline 1 a 12 & $\begin{array}{c}\text { PA13 } \\
\text { (de } 13,00 \text { a } 13,99 \text { ) }\end{array}$ & 511 & 1407 & 0 & 0 & 966 & 528 & 0 & 0 & 4,897 & 13,48 & 15,0 \\
\hline $1 \mathrm{a} 12$ & $\begin{array}{c}\text { PA14 } \\
\text { (de } 14,00 \text { a } 14,99 \text { ) }\end{array}$ & 1124 & 2403 & 0 & 0 & 966 & 1364 & 1090 & 552 & 4,633 & 14,25 & 15,0 \\
\hline 1 a 12 & $\begin{array}{c}\text { PA15 } \\
\text { (de } 15,00 \text { a } 15,99 \text { ) }\end{array}$ & 675 & 405 & 0 & 0 & 966 & 1364 & 1090 & 0 & 4,264 & 15,48 & 15,0 \\
\hline Ratio & & 14,0 & 11,5 & 13,5 & 14,5 & 15,5 & 16,5 & 17,5 & 18,5 & 4,264 & 15,48 & 15,0 \\
\hline Acidez & & 4,714 & 5,739 & 4,889 & 4,552 & 4,258 & 4,000 & 3,771 & 3,568 & 4,264 & 15,48 & 15,0 \\
\hline
\end{tabular}

Tabela 6. Resultado do plano de mistura para $\Gamma=7$ gerado pelo modelo apresentado na seção 3 - as três últimas colunas consideram que o desvio na acidez da base será de $2 \%$.

\begin{tabular}{|c|c|c|c|c|c|c|c|c|c|c|c|c|}
\hline \multirow{3}{*}{ Mês } & & \multicolumn{8}{|c|}{ (consumo esperado de precoce: máximo de $15 \%$ ) } & \multirow[t]{2}{*}{ desvio: } & \multicolumn{2}{|l|}{$2 \%$} \\
\hline & & Precoce & BA11 & BA13 & BA14 & BA15 & BA16 & BA17 & BA18 & & & \\
\hline & & \multicolumn{8}{|c|}{ Quantidade em toneladas } & Acidez & Ratio & $\%$ Precoce \\
\hline 1 a 12 & $\begin{array}{c}\text { PA11 } \\
\text { (de } 11,00 \text { a 11,99) }\end{array}$ & 0 & 2613 & 0 & 0 & 0 & 0 & 0 & 0 & 5,854 & 11,27 & $0,0 \%$ \\
\hline 1 a 12 & $\begin{array}{c}\text { PA13 } \\
\text { (de } 13,00 \text { a } 13,99 \text { ) }\end{array}$ & 511 & 1407 & 0 & 0 & 966 & 528 & 0 & 0 & 4,981 & 13,25 & $15,0 \%$ \\
\hline 1 a 12 & $\begin{array}{c}\text { PA14 } \\
\text { (de } 14,00 \text { a 14,99) }\end{array}$ & 1124 & 2403 & 0 & 0 & 966 & 1364 & 1090 & 552 & 4,711 & 14,01 & $15,0 \%$ \\
\hline 1 a 12 & $\begin{array}{c}\text { PA15 } \\
\text { (de } 15,00 \text { a } 15,99)\end{array}$ & 675 & 405 & 0 & 0 & 966 & 1364 & 1090 & 0 & 4,335 & 15,23 & $15,0 \%$ \\
\hline Ratio & & 14,0 & 11,5 & 13,5 & 14,5 & 15,5 & 16,5 & 17,5 & 18,5 & & & \\
\hline Acidez & & 4,714 & 5,854 & 4,987 & 4,643 & 4,343 & 4,080 & 3,847 & 3,639 & & & \\
\hline
\end{tabular}

especificação de ratio para o produto "PA14" é 14,00 e o valor resultante do plano de mistura será 14,01 .

A análise desses resultados evidencia que a abordagem de otimização robusta para tratar incertezas associadas aos parâmetros de acidez do suco oferece elementos importantes para dar apoio ao processo de tomada de decisão no planejamento da produção de suco concentrado congelado de laranja. Isso ocorre principalmente no que tange à elaboração do plano de mistura e na política de estoques para compensar incertezas na acidez do suco. Convém salientar que outras análises poderiam ter sido feitas considerando-se incertezas em outros parâmetros do modelo, por meio de abordagens de otimização robusta similares. 0 leitor interessado pode consultar Munhoz (2009) para outra análise desse mesmo problema utilizando outro modelo de programação linear robusto com incertezas na demanda dos produtos acabados.

\section{Conclusões e perspectivas}

Neste trabalho apresentou-se uma abordagem de otimização linear robusta para o planejamento agregado de produção na indústria cítrica, que admite incertezas nos parâmetros de acidez das bases de suco. Essa abordagem considera que a distribuição de probabilidade de acidez não precisa ser conhecida, mas apenas simétrica dentro de um intervalo de variação definido pelo tomador de decisão. llustra-se como esse modelo oferece a flexibilidade de proteger o plano de produção contra incertezas no referido parâmetro. Tal flexibilidade se traduz na possibilidade de variar-se o parâmetro de incerteza $\Gamma$, cuja função é ajustar a robustez do modelo proposto contra 0 nível de conservadorismo da solução. Os resultados obtidos mostram tal comportamento: quanto maior a proteção contra a probabilidade de violação das restrições que contêm os parâmetros com incertezas, maior será o custo total da solução gerada pelo modelo. Com isso, pode-se analisar de forma mais objetiva 0 trade-off existente entre a robustez e a qualidade da solução. Como os modelos envolvidos são lineares, os tempos de processamento para resolvê-los pelo software GAMS/CPLEX são da ordem de poucos segundos, não sendo, portanto, um limitante para a aplicação dessa abordagem na prática. 
0 presente trabalho oferece várias oportunidades para pesquisa futura. Por exemplo, seria interessante incorporar no presente modelo de otimização robusta outras características que interferem na determinação da especificação do ratio da fruta, tais como a região produtora e a florada. Além disso, em Munhoz (2009) também foi apresentada outra abordagem de otimização robusta similar à do presente trabalho, mas que considera incerteza na demanda dos produtos acabados. Seria interessante combinar as duas abordagens de otimização robusta e comparar os resultados da abordagem combinada com abordagens baseadas em restrições de chance e com abordagens baseadas em lógica fuzzy. Também seria interessante estudar a incorporação da demanda dos produtos acabados (conjunto de restrições (A4)) à matriz tecnológica, utilizando-se uma variável artificial $x_{n+1}=1$ neste conjunto de restrições e variando-se $\Gamma$ entre 0 e 1, conforme em Li e lerapetritou (2008).

Outra perspectiva interessante para pesquisa futura seria incluir no presente modelo de planejamento agregado de produção o planejamento de distribuição do produto acabado, pois, nesse setor, os sistemas de distribuição de produtos em geral são internacionais, com armazéns de distribuições em diferentes geografias, tais como EUA, Europa e Ásia. Em Munhoz (2000) e Munhoz e Morabito (2001a, b) pode ser encontrada uma descrição de um sistema de distribuição de suco de laranja concentrado de laranja de forma simplificada. Outra investigação futura importante seria desenvolver procedimentos para desagregação dos planos agregados gerados pelas abordagens de otimização robusta. Dessa forma, seria possivel mostrar como esses modelos táticos de planejamento agregado poderiam ser desdobrados em planos operacionais, que por sua vez teriam os seus resultados controlados e também poderiam retroalimentar os planos táticos.

\section{Referências}

AL-SHAMMARI, M.; DAWOOD, 1. Linear programming applied to a production blending problem: a spreadsheet modeling approach. Production and Inventory Management Journal, v. 38, n. 1, p. 1-7, 1997.

ARENALES, M. N. et al. Pesquisa Operacional. Rio de Janeiro: Editora Campus; Elsevier, 2007.

AXSATER, S.; JONSSON, H. Aggregation and disaggregation in hierarchical production. European Journal of Operational Research, n. 17, p. 338-350, 1984. http:// dx.doi.org/10.1016/0377-2217(84)90129-2

BAZARAA, M. S.; JARVIS, J. J.; SHERALl, H. D. Linear Programming and Network Flows. John Wiley \& Sons, lnc., 1990.

BERTSIMAS, D.; SIM, M. Robust discrete optimization and network flows. Mathematical Programming Series B, v. 98, n. 1-3, p. 49-71, 2003. http://dx.doi.org/10.1007/ s10107-003-0396-4
BERTSIMAS, D.; SIM, M. The price of robustness. Operations Research, v. 52, n. 1, p. 35-53, 2004. http://dx.doi. org/10.1287/opre.1030.0065

BERTSIMAS, D.; THIELE, A. A robust optimization approach to inventory theory. Operations Research, v. 54, n. 1, p. 150-168, 2006. http://dx.doi.org/10.1287/ opre. 1050.0238

BROOKE, A.; KENDRICK, D.; MEERAUS, A. Release 2.25 GAMS - A User's Guide.San Francisco:The Scientific Press, 1992.

CAIXETA FILHO, J. V. Estrutura de modelagem para programação de colheita de laranja. 1993. Tese (Doutorado em Engenharia de Transportes)-Universidade de São Paulo,São Paulo, 1993.

CAIXETA FILHO, J. V. Orange harvesting scheduling management: a cases tudy. Journal of the Operational Research Society, v. 57, p. 637-642, 2006. http://dx.doi. org/10.1057/palgrave.jors.2602041

CASTILHO, F. T. Organização dos produtores para a comercialização da laranja: o caso do pool de produtores de Matão-SP. 2006. Dissertação (Mestrado em Engenharia de Produção)-Universidade Federal de São Carlos, São Carlos, 2006.

FOOD AND AGRICULTURE ORGANIZATION OF THE UNITED NATIONS - FAO. Oranges production. Disponívelem: $<$ http://faostat.fao.org>. Acesso em: 01 maio 2009.

GEOFFRION, A. M. A priori error bounds for procurement commodity aggregation in logistics planning models. Naval Research Logistics Quarterly, v. 24, n. 2, p. 201-212, 1977. http://dx.doi.org/10.1002/ nav.3800240202

HASSE, G. A laranja no Brasil 1500-1987: a história da agroindústria cítrica brasileira, dos quintais coloniais à fábricas exportadoras de suco do século XX. São Paulo: Duprat \& lobe Propaganda, 1987.

HAX, A. C.; MEAL, H. C. Hierarchical integration of production planning and scheduling. In: GEISLER, M. A. (Ed.). Studies in Management Sciences. New York: North Holland-American Elsevier, 1975. v. 1 Logistics.

INSTITUTO BRASILEIRO DE GEOGRAFIA E ESTATÍSTICA - IBGE. Levantamento Sistemático da Produção Agrícola. IBGE, 2010. Disponível em: $<$ http://www.ibge.gov.br/home/estatistica/indicadores/ agropecuaria/lspa/lspa_201004_5.shtm>. Acesso em: 01 jun. 2010.

JOHNSON, L. A.; MONTGOMERY, D. C. Operations Research in Production Planning, Scheduling, and Inventory Control. New York: John Wiley \& Sons, Inc., 1974.

Ll, Z.; IERAPETRITOU, M. G. Robust optimization for process scheduling under uncertainty. Industrial \& Engineering Chemestry Research, v. 47, p. 4148-4157, 2008. http:// dx.doi.org/10.1021/ie071431u

MUNHOZ, J. R. Um modelo baseado em programação linear e programação de metas para análise de um sistema de produção e distribuição de suco concentrado congelado de laranja. 2000. Dissertação (Mestrado em Engenharia de Produção)-Universidade Federal de São Carlos, São Carlos, 2000.

MUNHOZ, J. R. Otimização no planejamento agregado de produção em indústrias de processamento de suco concentrado congelado de laranja. 2009. Tese (Doutorado em Engenharia de Produção)-Universidade Federal de São Carlos, São Carlos, 2009. 
MUNHOZ, J. R.; MORABITO, R. Um modelo baseado em programação linear e programação de metas para análise de um sistema de produção e distribuição de suco concentrado congelado de laranja. Gestão \& Produção, v. 8, n. 2, p. 139-159, 2001a.

MUNHOZ, J. R.; MORABITO, R. A goal programming model for frozen concentrated orange juice production and distribution system. Opsearch, v. 38, n. 6, p. 630-646, 2001b.

MUNHOZ, J. R.; MORABITO, R. Otimização no planejamento agregado de produção em indústrias de processamento de suco concentrado congelado de laranja. Gestão \& Produção, v. 17, n.3, p. 465-481, 2010. http://dx.doi. org/10.1590/S0104-530X2010000300003

NAHMIAS, S. Production and Operations Analysis. 6. ed. McGraw-Hill, 2009.

NEVES, M. F. et al. Mapeamento e quantificação do sistema agroindustrial citrícola. In: WORKSHOP DO SISTEMA AGROINDUSTRIAL CITRÍCOLA, 2004, Ribeirão Preto. Anais... Ribeirão Preto: USP, 2004. Relatório parcial. Disponível em: <http://www.pcarp.usp.br/acsi/ anterior/808/newpage6.htm>.Acesso em: 12 maio 2008.

PINTO, K. C. R. Contribuição à análise de decisão sobre os sistemas de distribuição física de suco de laranja brasileiro de exportação. 1996. Tese (Doutorado em Engenharia de Transportes)-Escola de Engenharia de São Carlos, Universidade de São Paulo,São Carlos, 1996.

SHAPIRO, J. F. Mathematical programming models and methods for production planning and scheduling. In: GRAVES, S. C.; RINNOOY KAN, A. H. G.; ZIPKIN, P. H. (Eds.). Logistic of Production and Inventory. Elsevier Science Publishers B. V., 1993. p. 371-439. (Handbooks in Operations Research and Management Science: v. 4).
SHAPIRO, J. F. Modeling the Supply Chain. Pacific Grove: Editora Duxbury, 2001.

SIM, M. Robust optimization. Tese (Doutorado)-Sloan School of Management, Massachusetts Institute of Technology, 2004.

SOYSTER, A. L. Convex programming with set-inclusive constraints and applications to inexact linear programming. Operations Research, v. 21, n. 1, p. 1154-1157, 1973. http://dx.doi.org/10.1287/ opre.21.5.1154

VIEGAS, F. C. P. et al. Processamento dos produtos cítricos com máquinas FMC. Araraquara: FMC do Brasil S. A., 1983. Apostila do Curso Processamento de laranja com máquinas FMC.

VIEIRA, A. C. A estratégia de verticalização da produção no setor citrícola. In: ENCONTRO NACIONAL DE ENGENHARIA DE PRODUÇÃO, 17., 1997, Gramado. Anais... ABEPRO, 1997. Disponível em: <http://www. abepro.org.br/biblioteca/enegep 1997_t3503.pdf>. Acesso em: 12 maio 2008.

WILlIAMS, H. P. Model Building in Mathematical programming. New York: John Wiley \& Sons, Inc.; 1978.

ZIPKIN, P. Exact and approximate cost function for product aggregates. Management Science, n. 28, p. 1002-1012, 1982. http://dx.doi.org/10.1287/ mnsc.28.9.1002

\section{Agradecimentos}

Os autores agradecem aos dois revisores anônimos pelos úteis comentários e sugestões. Esta pesquisa contou com apoio parcial do CNPq e Fapesp.

\title{
A robust optimization approach for the aggregate production planning in the citrus industry
}

\begin{abstract}
In this work, a frozen concentrated orange juice aggregate production planning is modeled taking into account uncertainty in some parameters. This generates an effective tool to support decision making. The robust optimization approach is based on a linear programming model with multiple products, stages and periods proposed by Munhoz and Morabito (2010). Besides decisions in the production, blending and storage of juices, the model also includes an orange harvesting plan, which considers orange maturation curves. A case study was developed in an orange juice company located in the State of Sao Paulo. This company has different facilities and a worldwide distribution system. The computational results obtained from this robust optimization approach, using an algebraic modeling language and a state-of-the-art optimization solver; indicate that the approach can be useful in real situations.
\end{abstract}

\section{Keywords}

Robust optimization. Production planning with multiple products. Stages and periods. Linear programming. Frozen concentrated orange juice. 
Anexo 1. Modelo determinístico de programação linear.

Função objetivo

$Z=\min \sum_{f} \sum_{g} \sum_{t} \sum_{j}$ CustoQL $_{f g t} \times Q L_{f g j t}+\sum_{f} \sum_{t}$ CustoQLH $_{f t} \times Q L H_{f t}+\sum_{t} \sum_{i}\left(\right.$ CustoHS $_{i t} \times H S_{i t}+$ CustoFS $\left._{i t} \times F S_{i t}\right)+$

$\sum_{t}\left(\right.$ CustoHH $_{t} \times \mathrm{HH}_{t}+$ CustoFH $\left._{t} \times \mathrm{FH}_{t}\right)+\sum_{t} \sum_{j}\left(\right.$ CustoHB $_{j t} \times H B_{j t}+$ CustoFB $\left._{j t} \times F B_{j t}\right)$

A função objetivo (A1) é composta pela soma do custo total da matéria-prima; do custo total de armazenagem de suco, base e precoce, e do custo total de falta de suco, base e precoce.

Restrições

Restrição de o nível total de processamento desse planejamento tático respeitar (planejamento hierárquico) a definição estratégica de nível de processamento:

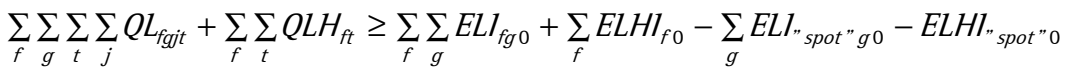

Restrições de níveis máximo e mínimo de processamento industrial:

$C P L_{t} \leq \sum_{f} \sum_{g} \sum_{j} Q L_{f g j t}+\sum_{f} Q L H_{f t} \leq C P H_{t} ; \forall t$

Equações de balanceamento de material:

$Q S_{i t}-$ Demanda $_{i t}+H S_{i(t-1)}-F S_{i(t-1)}-H S_{i t}+F S_{i t}=0 ; \forall i, t$

$Q H P_{t}-\sum_{j} \sum_{i} Q H_{j t i}+H H_{(t-1)}-F H_{(t-1)}-H H_{t}+F H_{t}=0 ; \forall t$

$Q B P_{j t}-\sum_{i} Q B_{j t i}+H B_{j(t-1)}-F B_{j(t-1)}-H B_{j t}+F B_{j t}=0 ; \forall j, t$

Equações de mistura para obtenção dos produtos finais conforme especificações a partir de bases e precoces:

$\frac{\left[\sum_{j}\left(Q B_{j t i} \times A B_{j}+Q H_{j t i} \times A H\right)\right]}{A S H_{i}} \leq \sum_{j}\left(Q B_{j t i}+Q H_{j t i}\right) ; \forall i, t$

$\frac{\left[\sum_{j}\left(Q B_{j t i} \times A B_{j}+Q H_{j t i} \times A H\right)\right]}{A S L_{i}} \geq \sum_{j}\left(Q B_{j t i}+Q H_{j t i}\right) ; \forall i, t$

$Q H_{j t i} \leq$ Porcentagem $_{j t} \times\left(Q B_{j t i}+Q H_{j t i}\right) ; \forall j, i, t$

$Q S_{i t}=\sum_{j}\left(Q B_{j t i}+Q H_{j t i}\right) ; \forall i, t$

Equações de relação de quantidade de laranja gerando quantidades de bases e precoces, incorporando o fator disponibilidade de fruta nos cálculos:

$Q L_{f g j t} \leq E L I_{f g 0} \times D L_{f g j t} ; \forall f, g, j, t$

$Q L H_{f t} \leq E L H I_{f 0} \times D H_{f t} ; \forall f, t$

$\sum_{f} \sum_{g} Q L_{f g j t}-Q B P_{j t} \times$ Rendimento $B_{j t}=0 ; \forall j, t$

$\sum_{f} Q L H_{f t}-Q H P_{t} \times$ Rendimento $H_{t}=0 ; \forall t$

Equações de balanceamento de material para fornecimento de laranja:

$E L_{f g t}-E L_{f g(t-1)}+\sum_{j} Q L_{f g j t}=0 ; \forall f, g, t$

$E L H_{f t}-E L H_{f(t-1)}+Q L H_{f t}=0 ; \forall f, t$

Restrição de capacidade de fornecimento de laranja:

$\sum_{j} \sum_{g} Q L_{f g j t}+Q L H_{f t} \leq C F_{f t} ; \forall f, t$ 